\title{
Acoustic evaluation of HEMA polymer gel dosimeter phantoms
}

\begin{abstract}
A new method for the evaluation of radiotherapy 3D polymer gel dosimeters has been developed using ultrasound to assess the significant structural changes that occur following irradiation of the dosimeters. Polymer gel dosimeters were being fabricated using a monomer named 2-Hydroxyl-Ethyl-Meta-Acrylate (HEMA) with the presence of gelatine as a gelling agent. The readymade gel which is the concentration for HEMA fixed at 2, 4 and 5\% would then undergo an ultrasonic evaluation to test for the propagation of sound speed through it. In the observation of relationship between the ultrasound propagation speeds as the doses increase (focusing at the cross region from overlapped beams) and concentration of monomer, the propagation speed for all the three polymer gel dosimeter phantoms still varies between 1460 to $1570 \mathrm{~m} / \mathrm{s}$ which is still in the range of speed of sound for human tissue [3]. The ultrasonic absorption attenuation coefficient dose sensitivity for polymer gel dosimeters for 2,4 and $5 \%$ of monomer are in the range of 0.02 to $0.6 \mathrm{~dB}$ which is equivalent to human tissue. As a comparison, it can be seen that gel phantoms with high concentration of monomer $(5 \%)$ is more sensitive to the radiation compared to the lower ( $2 \%$ and $4 \%$ ) concentrations. Regarding the absolute results of mechanical and acoustic properties; the copolymer-in-oil phantom is equivalent with soft tissue.
\end{abstract}

Keyword: Polymer gel; Radiotherapy; Ultrasound 